\title{
Organ-level vascularization: The Mars mission of bioengineering
}

\author{
Boyang Zhang, $\mathrm{PhD},{ }^{\mathrm{a}, \mathrm{b}}$ and Milica Radisic, $\mathrm{PhD}^{\mathrm{c}, \mathrm{d}, \mathrm{e}, \mathrm{f}}$
}

\section{ABSTRACT}

Organ-level vascularization has been a long-standing challenge in the field of tissue engineering. Recent advances, particularly in the use of projection stereolithography and food colors as photoabsorbers are highlighted, as are several recent studies on the clinical translation of engineered vasculature. (J Thorac Cardiovasc Surg 2020;159:2003-7)

Engineering large-scale functional tissues and organs for tissue repair or organ replacement has always been the ultimate goal of tissue engineering and regenerative medicine. This grand challenge, which is as old as the field itself, is so complex and daunting that it is fair to view it as the Mars mission of bioengineering. Although there are numerous challenges and hurdles that we have to overcome in this process, the problem is worth solving because the clinical issues of organ shortage and endstage diseases will not go away. The move toward a solution is inevitable and will fundamentally expand the arsenal of modern medicine from the use of drugs to the use of engineered tissues. As with any great strides in science, in the process of tackling this grand challenge in tissue engineering, the advances we have seen over the years are already benefiting and opening up new fields, such as organ-on-a-chip engineering, biorobotics, and drug delivery.

\section{EVOLUTION OF TISSUE VASCULARIZATION AND ORGAN-LEVEL VASCULARIZATION}

At the heart of this problem of constructing large functional tissues or organs is tissue vascularization, which is relevant for all organ systems, but especially in cardiac tissue engineering. The high metabolic rate of cardiomyocytes is reflected by the capillary density in the heart:

\footnotetext{
From the ${ }^{\mathrm{a}}$ Department of Chemical Engineering and ${ }^{\mathrm{b}} \mathrm{School}$ of Biomedical Engineering, McMaster University, Hamilton, Ontario, Canada; ${ }^{\mathrm{c} D e p a r t m e n t}$ of Chemical Engineering and Applied Chemistry and ${ }^{\mathrm{d}}$ Institute for Biomaterials and Biomedical Engineering, University of Toronto, Toronto, Ontario, Canada; ${ }^{\mathrm{e}}$ Toronto General Research Institute, University Health Network, Toronto, Ontario, Canada; and ${ }^{\mathrm{f}}$ The Heart and Stroke/Richard Lewar Centre of Excellence, Toronto, Ontario, Canada.

Supported by the Canadian Institutes of Health Research (CIHR) (Operating Grant Nos. MOP-126027 and MOP-137107), the National Sciences and Engineering Research Council of Canada (NSERC) (Discovery Grant No. RGPIN 32698210), an NSERC-CIHR Collaborative Health Research Grant (No. CHRP 493737 16), a NSERC Strategic Grant (No. STPGP 506689-17), and a National Institutes of Health grant (No. 2R01 HL076485) to Dr Radisic as well CIHR Operating Grant
}

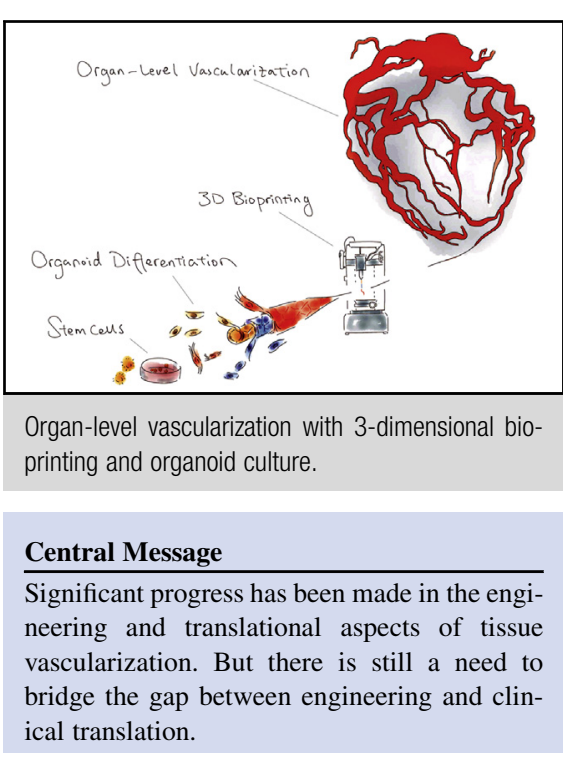

This Invited Expert Opinion provides a perspective on the following paper: Science. 2019;364:458-464. https://doi.org/10.1126/ science.aav9750.

See Commentaries on pages 2008 and 2010.

Almost every cardiomyocyte neighbors a capillary to facilitate efficient mass transfer. ${ }^{1}$ Establishing functional perfusable vasculature is a fundamental challenge we need to overcome for both the long-term goal of building an entire transplantable organ in the lab and the mediumterm goal of repairing large tissue defects by implanting engineered tissues. Initial vascularization solutions stemmed from biological methods (ie, growth factor

No. MOP-408286 and NSERC Discovery Grant No. RGPIN-2018-05500 to Dr Zhang.

Editorial review was handled by Associate Editor Francis D. Pagani, MD, PhD

Received for publication July 3, 2019; revisions received Aug 2, 2019; accepted for publication Aug 10, 2019; available ahead of print Oct 23, 2019.

Address for reprints: Boyang Zhang, $\mathrm{PhD}$, Department of Chemical Engineering, McMaster University, 1280 Main Street West, Hamilton, Ontario, L8S 4L8,

Canada (E-mail: zhangb97@mcmaster.ca); and Milica Radisic, PhD, Department of Chemical Engineering and Applied Chemistry, University of Toronto, 200 College Street, Toronto, Ontario M5S 3E5, Canada (E-mail: m.radisic@ utoronto.ca).

$0022-5223 / \$ 36.00$

Copyright (c) 2019 by The American Association for Thoracic Surgery

https://doi.org/10.1016/j.jtcvs.2019.08.128 
delivery, gene therapy, and cell therapy) that attempted to stimulate endogenous blood vessel growth into the infarcted myocardium, reducing the expansion of the infarct and improving heart function. ${ }^{2}$ But these approaches that seek to improve vascular supply in preexisting organs with vascular insufficiency have met with limited success. For example, the delivery of angiogenic growth factors such as vascular endothelial growth factor by protein or gene therapy can result in vasculature that is highly disorganized and leaky. ${ }^{3}$ This approach is further limited by complex pharmacokinetic parameters, the high cost to maintain growth factor levels, the requirement for localized effects and low in vivo transfection rates in gene therapy. ${ }^{4}$ A relatively new branch of research focuses on manipulating endogenous noncoding RNAs $(\sim 22 \mathrm{nu}-$ cleotides), known as microRNAs, to direct myocardial angiogenesis, the early results of which are promising. ${ }^{5}$ However, it seems unlikely that endogenous endothelial repair alone can regenerate an injury as large as a major myocardial infarct. ${ }^{6}$ The natural development of vasculature and tissue takes place at a time and physical scale that is too slow and too small to satisfy the level of therapeutic support that clinicians seek in dealing with large tissue defects and end-stage disease. Therefore, there is a need to engineer functional tissues in vitro before implantation to repair large tissue defects. However, engineered thick tissue construct cannot be viably maintained in the lab or in vivo unless there is perfused vasculature, which is also difficult to establish with the use of growth factor alone. This challenge leads to the value proposition of tissue engineering, which seeks to deliver a therapeutic alternative with engineering methods to overcome this limitation.

Engineering strategies to accelerate vascularization include the direct fabrication of branching templates on which endothelial cells can assemble in vitro. Borenstein and colleagues ${ }^{7}$ and Zhang and colleagues ${ }^{8}$ demonstrated the fabrication of vascular network templates by silicon etching using polydimethylsiloxane molding. However, materials such as polydimethylsiloxane are nonbiodegradable and thus have limited usefulness in vivo. Since then, the field has shifted to the use of biocompatible and biodegradable materials such as polylactide-co-glycolide and polyglycerol sebacate. The mechanical strength and the biodegradable properties of polyglycerol sebacate allow it to be molded into a vascular network using standard soft lithography techniques. ${ }^{9}$ A subtractive method is often used, where a sacrificial vascular network is first created from gelatin and then embedded within a cell-laden hydrogel. ${ }^{10,11}$ The gelatin structures are subsequently removed by increasing the temperature of the construct to physiological conditions, leaving behind open channels for endothelialization. Later on, mechanically stable carbohydrate glass or pluronic acid, which can be printed with a 3-dimensional (3D) printer, were used to replace gelatin as the sacrificial material, which greatly facilitated the scaling of this subtractive fabrication method. ${ }^{12}$ However, creating complex biological structures by printing the sacrificial material in midair is still challenging. Nonetheless, this is a popular technique, especially in fabricating microphysiological models.

More recently, rapid advances in hydrogel fabrication have been made to unlock the design freedom in organ engineering. Miller and colleagues ${ }^{13}$ recently demonstrated the 3D printing of ultracomplex organ-level intravascular and multivascular systems in photopolymerizable hydrogel with projection stereolithography (Figure 1,A). Specifically, perfusable 3D networks resembling a vascularized lung alveolar sac was printed and then perfused with blood, which revealed that the redirection of blood flow and intravascular mixing induced by alveolar inflation may have improved the rate of oxygen exchange (Figure $1, B$ ). This observation points to tangible organlevel functional improvements as a result of recapitulating organ-level biological architectures. Miller and colleagues ${ }^{13}$ were able to create complex curved shapes, entangled vascular networks, and shapes identical to 3D bicuspid valves, using the simplest materials: photocrosslinkable polyethylene glycol and commercial food dyes that served as photoabsorber additives for projection stereolithography. Their study is unprecedented in terms of both resolution and complexity of the created shapes. The team also demonstrated that implantation of vascularized liver tissue created by this approach is possible. In the future, if integrated with 3D organoid culture, unprecedented biological complexity and scale could be simultaneously established.

Another approach of creating complex 3D organ-level structures is based on freeform reversible embedding of suspended hydrogels. ${ }^{14}$ Printing biomaterial in midair has always been a major challenge, especially for slow-gelling materials. To overcome this issue, the freeform reversible embedding of suspended hydrogels technique allows biological materials to be printed in a bath of viscous gel, which can serve as a temporary, thermoreversible, and biocompatible support. This approach opens up the possibility of directly printing natural hydrogels, such as collagen, fibrin, Matrigel (Corning, NY), or even decellularized matrices into complex 3D structures. Recently, as a proof-of-concept study, the technique was used to print cell-laden decellularized hydrogels into the anatomical structure of an entire heart, including the heart chambers and the major coronary vessels. Although tissue function is yet to be demonstrated at this organ-scale construct, the high level of structural complexity of the 3D printed natural biomaterial achieved is a major advance. 
3D printed organ-level vasculature

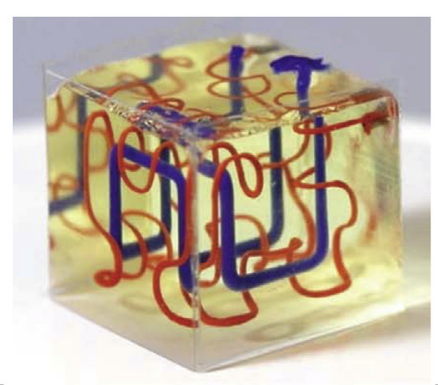

A

B

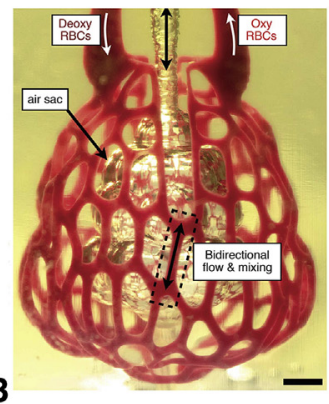

IPSC derived vascular organoids
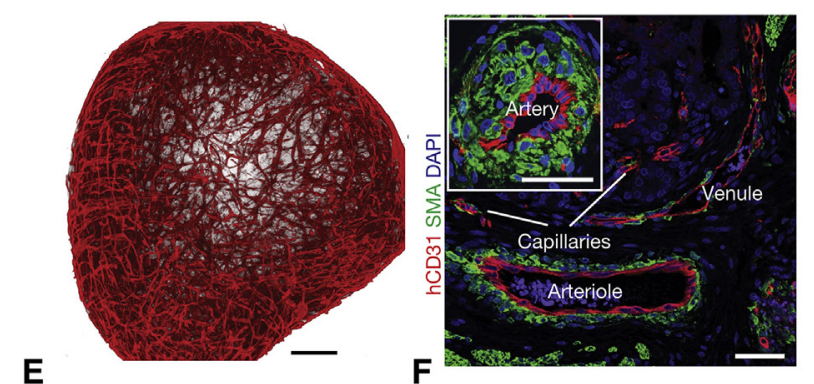

Pre-organized vasculature accelerate in vivo vascularization
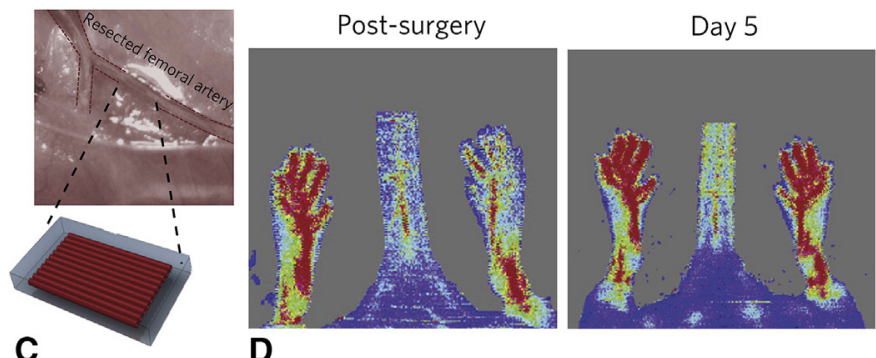

D engineering vascular networks

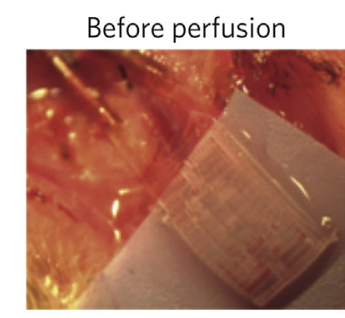

G
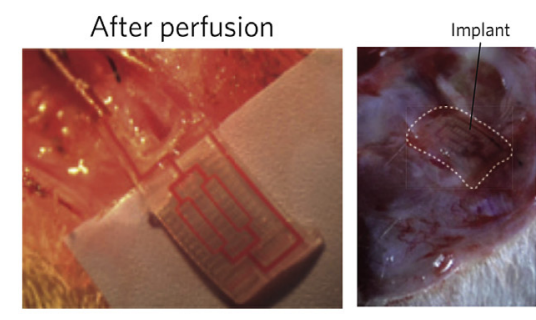

H

FIGURE 1. Examples of engineering tissue and organ vascularization techniques. A, 3-Dimensional printed hydrogels (20 wt \% polyethylene (glycol) diacrylate, $6 \mathrm{kDa}$ ) with interpenetrating Hilbert curves. B, Photograph of a 3-dimensional printed hydrogel embedded with a vascularized lung alveolar subunit. Red blood cells were perfused while the air sac was ventilated (scale bar, $1 \mathrm{~mm}$ ). C, Illustration of the tissue graft with aligned vasculature and implantation site within the cauterized femoral artery space. D, Laser Doppler imaging of distal limbs over time showing vascular perfusion established by day 5 postsurgery. E, 3-Dimensional reconstruction of an induced pluripotent stem cells-derived vascular organoid (CD31 red). F, Development of arteries, arterioles, capillaries, and venules in transplanted human organoids shown by staining for hCD31 and smooth muscle actinin. G, Surgical connection of engineered vascular scaffold (AngioChip) showing immediate vascular perfusion after the surgical procedure. H, Image of vascular implant 1 week after implantation. All images are adapted with permission. IPSC, Induced pluripotent stem cells.

Much progress has been made since the first publication of microfluidic soft hydrogel with the conventional microfabrication techniques in 2007. ${ }^{15}$ Today there is no shortage of engineering methods to construct complex 3D hydrogel structures. However, being able to precisely pattern cells and lay down complex organ-level architecture, although important, is only the first step of a long journey to establishing tissue or organ functions at the therapeutic level. The major shortcomings of the 3D printing hydrogelbased methods are the inability to simultaneously reach physiological cell density within the gel while preserving the engineered vasculature as well as the inability to easily apply this architecture in vivo, especially in high-pressure environments. Properties such as burst strength and suture retention strength are generally inferior in hydrogels, compared with the polymers used to construct commonly used vascular grafts.

Tissue remodeling is a crucial step in achieving high physiological cell density and tissue maturation. Because the dynamic nature of the tissue remodeling process, there is a need to guide tissue growth at multiple length and time scales that could potentially be coordinated by the choice of biomaterials or combination of multiples biomaterials. Multimaterial extrusion-based printing of vascularized cartilage tissues was demonstrated, which showed tissue remodeling and differentiation can be supported in large tissue constructs with multimaterial-based $3 \mathrm{D}$ printing approach. ${ }^{16}$ However, multimaterial printing of complex perfusable vascular structure is yet to be demonstrated and might be necessary to further elevate biological functionality and structural stability of printed tissues.

To date, the entire tissue engineering field is mainly rooted in enhancing the precision of scaffold and hydrogel fabrication, where the material still functions mainly as static structural support and lacks dynamic functionalities. Appreciating the complex and dynamic nature of human tissues, this field might require innovative tissue assembly strategies that do not merely view a scaffold as a static skeleton, but a dynamic machine that guides tissue assembly over time. Recognizing this fourth dimension in biofabrication could transform the way we approach tissue and organ engineering. For instance, 4-dimensional printing 
and origami tissues are emerging developments that combine $3 \mathrm{D}$ printing or micropatterning with smart functional biomaterials that can change shape over time, based on their intrinsic properties or external stimulation. ${ }^{17}$ Although the technique is more widely used in developing soft-robotics, sensors, and actuators, novel tissue engineering solutions based on this concept are still emerging.

\section{CLINICAL TRANSLATION OF ENGINEERED VASCULATURE}

As challenging as engineering organ-level vasculature, establishing rapid vascular integration and maturation in vivo is crucial in maintaining the viability and growth of the implanted vessels and tissues posttransplantation. By simply incorporating endothelial cells in an engineered tissue implant, blood vessels can naturally form in vivo from the self-assembly of the implanted endothelial cells, but vascular perfusion will take 1 to 2 weeks to establish. ${ }^{18}$ During this period, implanted parenchymal cells, such as cardiomyocytes, will die within 1 to 2 days postimplantation without the supply of oxygen and nutrients from vascular perfusion. The vascularization process is slow because the randomly distributed endothelial cells have to come together to assemble into mature vasculature. By preorganizing the endothelial cells in vitro into an array of linear vessels in parallel, the time to achieve vascular perfusion in vivo posttransplantation can be significantly reduced to just 3 to 5 days (Figure 1, $C$ and $D$ ). ${ }^{19,20}$ When the engineered vasculature is accompanied by parenchymal cells, such as hepatocytes or pancreatic islets that are highly susceptible to apoptosis due to insufficient oxygen and nutrient diffusion, the resulting accelerated vascularization led to significant functional and therapeutic improvements in vivo. ${ }^{20}$

Long-term vascular maturation and the prevention of vascular regression are also key issues to consider. Although blood vessels resulting from primary human endothelial cells alone were demonstrated to be capable of rapid vascular integration, their long-term vascular maturation will require the participation of relevant stromal cells. Furthermore, the formation of the vascular hierarchy will require the differentiation of arterial and venous phenotypes. These issues could potentially be addressed by taking advantage of the phenotypic plasticity of induced pluripotent stem cells (iPSC)-derived endothelial progenitor cells. iPSC-derived human blood vessel organoids have been shown to contain both endothelial cells and pericytes that can self-assemble into functional and matured capillary networks (Figure 1,E) ${ }^{21}$ When transplanted, the blood vessel organoids form stable, perfused vascular trees, including arteries, arterioles, and venules, in mice (Figure 1,F). The system can also be used to model diseases such as diabetic vasculopathy that is manifested by the thickening of the vascular basement membrane, which now can be directly observed in the organoid culture in vitro. It is also important to emphasize that the use of iPSC as a cell source will not only lead to personalized drug discovery, but also result in significantly improved biocompatibility and immunocompatibility when transplanted.

The reciprocating interaction of the vasculature and the parenchymal tissues, which are often developed and matured concurrently, also play a vital role in the organ development process. Tissue organoids produced by the triculture of iPSCderived hepatocytes with primary endothelial cells and mesenchymal stem cells were shown to not only improve in vivo vascularization, but the resulting vasculature can also help stimulate the maturation of iPSC-hepatocytes into matured tissues resembling the adult liver. ${ }^{22}$ This same strategy has also been shown to be applicable to a variety of organ systems. ${ }^{23}$ Furthermore, taking advantage of the plasticity of iPSC-derived endothelial cells, the co-culture of these endothelial cells with parenchymal cells could potentially induce organ-specific vascular differentiation, which should be explored in the future. ${ }^{24}$ Preorganized vasculature supported with parenchymal tissues and sourced from iPSCs or organoids can clearly provide in vivo vascularization and has already presented sufficient evidence of efficacy in small animals. Translation to large animals is potentially feasible but is yet to be determined. The challenge of vascularization strategies that relies on native angiogenesis and vascular integration is that the rate of vascularization and vascular maturation could drastically change in clinical translation due to the decrease in surface-to-volume ratio of the tissue implant as well as in the rate of vascular growth in humans, especially older adults. Therefore, it remains to be seen if prior success in small animals is translatable to human trials.

Establishing direct surgical vascular connection is feasible and could overcome this reliance on the rate of natural vascular integration. We reported the fabrication of a biodegradable scaffold (commonly referred to as AngioChip) that contains a perfusable, branched, 3D microchannel network coated with endothelial cells to mimic the

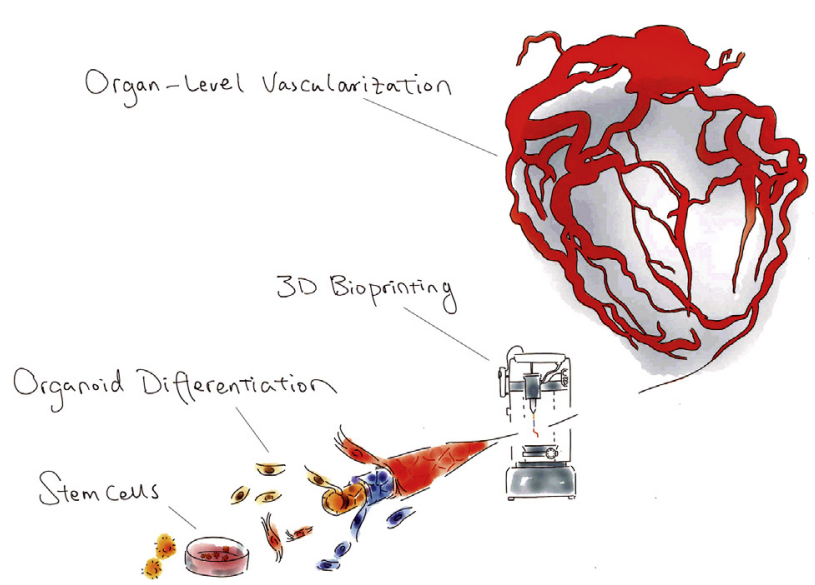

FIGURE 2. Organ-level vascularization with 3-dimensional bioprinting and organoid culture. 
human vascular networks. ${ }^{25}$ The permeability of the network was improved without compromising mechanical stability by incorporating nanopores and microholes in the vessels walls, which also permits the intercellular crosstalk and extravasation of monocytes and endothelial cells on biomolecular stimulation. Surrounding the vasculature, a variety of parenchymal cells can be cultured. We showed our engineered vascularized hepatic tissue can metabolize drugs delivered through the internal vasculature and our engineered vascularized cardiac muscles can achieve high physiological cell density and contract macroscopically without collapsing the internal networks. Lastly, we demonstrated direct surgical vascular anastomosis of our engineered tissue to the femoral vessels of rat hindlimbs, establishing immediate blood perfusion after surgical implantation (Figure 1, $G$ and $H$ ). However, a current limitation is that it relies on the scalability of the synthetic scaffolds. Furthermore, to engineer organ-level vasculature with surgical vascular connections, engineered branching vasculature will need to be more consistently assessed from standard properties that are used to assess the functionality of vascular grafts, including burst pressure, suture retention strength, platelet adhesion, complement activation, thrombogenicity, and long-term patency in vivo. Ultimately, the viability of the tissue implants will rely on sustained vascular perfusion, which is especially crucial during the early days postimplantation.

\section{FUTURE PERSPECTIVES}

Considering both engineering complexity and translational efficacy, it is clear that designing a functional tissue or an organ will require the right balance between tissue self-assembly and engineered design. This balance relies on advances in our bioengineering capabilities and our understanding of cell biology (Figure 2). Often it appears that there are so many techniques one can choose from and yet few of these methods can provide the complete tissue engineering solution that covers all the requirements. However, advances in bioengineering, even if they offer an incomplete solution, often lead to deeper understanding of cell biology. A more in-depth comprehension in cell biology will, in turn, guide the development of the next generation of engineering strategies. Meanwhile, to benefit from the current development in bioengineering, it is important to first acquire an understanding of the required level of tissue functionality, structural complexity, and material compatibility. Only then can the clinical application be tailored to the most appropriate engineering technique.

\section{Conflict of Interest Statement}

Drs Zhang and Radisic are co-founders of TARA Biosystems, Inc, and hold equity in this company.

The authors thank Dawn Lin for making Figure 2.

\section{References}

1. Brutsaert DL. Cardiac endothelial-myocardial signaling: its role in cardiac growth, contractile performance, and rhythmicity. Physiol Rev. 2003;83: 59-115.

2. Engel FB, Hsieh PC, Lee RT, Keating MT. FGF1/p38 MAP kinase inhibitor therapy induces cardiomyocyte mitosis, reduces scarring, and rescues function after myocardial infarction. Proc Natl Acad Sci U S A. 2006;103: 15546-51.

3. Jain RK, Au P, Tam J, Duda DG, Fukumura D. Engineering vascularized tissue. Nat Biotechnol. 2005;23:821-3.

4. Melly L, Boccardo S, Eckstein F, Banfi A, Marsano A. Cell and gene therapy approaches for cardiac vascularization. Cells. 2012;1:961-75.

5. Zangi L, Lui KO, von Gise A, Ma Q, Ebina W, Ptaszek LM, et al. Modified mRNA directs the fate of heart progenitor cells and induces vascular regeneration after myocardial infarction. Nat Biotechnol. 2013;31:898-907.

6. Sun L, Bai Y, Du G. Endothelial dysfunction — an obstacle of therapeutic angiogenesis. Ageing Res Rev. 2009;8:306-13.

7. Borenstein JT, Terai H, King KR, Weinberg EJ, Kaazempur-Mofrad MR, Vacanti JP. Microfabrication technology for vascularized tissue engineering. Biomed Microdevice. 2002;4:167-75.

8. Zhang B, Peticone C, Murthy SK, Radisic M. A standalone perfusion platform for drug testing and target validation in micro-vessel networks. Biomicrofluidics. 2013;7:44125.

9. Fidkowski C, Kaazempur-Mofrad MR, Borenstein J, Vacanti JP, Langer R, Wang Y. Endothelialized microvasculature based on a biodegradable elastomer Tissue Eng. 2005;11:302-9.

10. Golden AP, Tien J. Fabrication of microfluidic hydrogels using molded gelatin as a sacrificial element. Lab Chip. 2007;7:720-5.

11. Tang MD, Golden AP, Tien J. Fabrication of collagen gels that contain patterned, micrometer-scale cavities. Adv Mater. 2004;16:1345.

12. Miller JS, Stevens KR, Yang MT, Baker BM, Nguyen DH, Cohen DM, et al Rapid casting of patterned vascular networks for perfusable engineered threedimensional tissues. Nat Mater. 2012;11:768-74.

13. Grigoryan B, Paulsen SJ, Corbett DC, Sazer DW, Fortin CL, Zaita AJ, et al. Multivascular networks and functional intravascular topologies within biocompatible hydrogels. Science. 2019;364:458-64.

14. Hinton TJ, Jallerat Q, Palchesko RN, Park JH, Grodzicki MS, Shue HJ et al. Three-dimensional printing of complex biological structures by freeform reversible embedding of suspended hydrogels. Sci Adv. 2015;1: e1500758.

15. Choi NW, Cabodi M, Held B, Gleghorn JP, Bonassar LJ, Stroock AD. Microfluidic scaffolds for tissue engineering. Nat Mater. 2007;6:908-15.

16. Kang H-W, Lee SJ, Ko IK, Kengla C, Yoo JJ, Atala A. A 3D bioprinting system to produce human-scale tissue constructs with structural integrity. Nat Biotechnol. 2016;34:312-9.

17. Gladman AS, Matsumoto EA, Nuzzo RG, Mahadevan L, Lewis JA. Biomimetic 4D printing. Nat Mater. 2016;15:413-8.

18. Shandalov Y, Egozi D, Koffler J, Dado-Rosenfeld D, Ben-Shimol D, Freiman A et al. An engineered muscle flap for reconstruction of large soft tissue defects. Proc Natl Acad Sci U S A. 2014;111:6010-5.

19. Mirabella T, MacArthur JW, Cheng D, Ozaki CK, Woo YJ, Yang M, et al. 3D printed vascular networks direct therapeutic angiogenesis in ischaemia. Nat Biomed Eng. 2017;1:0083.

20. Baranski JD, Chaturvedi RR, Stevens KR, Eyckmans J, Carvalho B Solorzano RD, et al. Geometric control of vascular networks to enhance engineered tissue integration and function. Proc Natl Acad Sci U S A. 2013;110 7586-91.

21. Wimmer RA, Leopoldi A, Aichinger M, Wick N, Hantusch B, Novatchkova M et al. Human blood vessel organoids as a model of diabetic vasculopathy. Nature 2019;565:505-10.

22. Takebe T, Sekine K, Enomura M, Koike H, Kimura M, Ogaeri T, et al. Vascularized and functional human liver from an iPSC-derived organ bud transplant. $\mathrm{Na}$ ture. 2013;499:481-4.

23. Takebe T, Enomura M, Yoshizawa E, Kimura M, Koike H, Ueno Y, et al. Vascularized and complex organ buds from diverse tissues via mesenchymal celldriven condensation. Cell Stem Cell. 2015;16:556-65.

24. Lin DSY, Guo F, Zhang B. Modeling organ-specific vasculature with organ-on-achip devices. Nanotechnology. 2019;30:024002.

25. Zhang B, Montgomery M, Chamberlain MD, Ogawa S, Korolj A, Pahnke A, et al Biodegradable scaffold with built-in vasculature for organ-on-a-chip engineering and direct surgical anastomosis. Nat Mater. 2016;15:669-78. 